\title{
Körperliche Aktivität unter Berliner Schulkindern
}

\section{Ergebnisse der Berliner Studie »Gesundheit im Kindesalter (GIK II) 1994/95}

\author{
Von H. Hüttner, R. Dortschy, H. Heß, H. Kahl, K. Tietze und G. Röseler
}

\section{Zusammenfassung}

Die sportliche Aktivität Berliner Schüler der 7. bis 10. Klassen, untersucht an Haupt- und Gymnasialschülern, ist hoch und hat sich in den vergangenen zehn Jahren nur gering verändert. In beiden Schülergruppen dominieren die Jungen beim Sporttreiben. Hauptschüler nennen cine höhere Stundenzahl körperlicher Aktivitäten als Gymnasiasten, Gymnasialschüler führen eher sportliches Training mit hoher Intensität aus. Insgesamt geht im Altersverlauf der Umfang der körperlichen Aktivität zurück, bei Mädchen mehr als bei Jungen. Die wichtigsten Einflußfaktoren auf das Sporttreiben sind der eigene Spaß am Sport, die Integration in einen Sportverein und der Einfluß der Freunde, zum Teil auch des Vaters auf das Sporttreiben. Sporttreibende fühlen sich gesünder. Es wurden insgesamt 5292 Schüler untersucht.

\begin{abstract}
Summary
Physical activity among Berlin school children - results of the Berlin study "Health of Children " (GIK II) 1994/95

Physical activity of Berlin school children is high and did not change in the last ten years. In public elementary school (called »Hauptschule«) and high school (»Gymnasium") among 7. to 10 . graders, boys are physically more active than girls. Elementary school students have a higher extent of athletic activities, high school students train with higher intensity. Both genders reduce physical activity berween 7 . and 10 . grade, girls more than boys. The most important influencing factors on exercise are the own engagement in sports, the membership in a sports club and the influence of friends, peers and parents, especially the father. Regularly active children feel themselves healthier. A total of 5292 pupils were investigated.
\end{abstract}

Jugendliche sind in der Regel körperlich aktiv; die Bewegungsformen reichen vom Umhergehen, Radfahren, Tanzen bis zum harten sportlichen Training. Ein gewisses $\mathrm{Ma} \beta$ an Belastungsintensität vorausgesetzt, geht regelmäßige körperliche Aktivität im Kindes- und Jugendalter mit der Reduktion von Mortalität und Morbidität im Erwachsenenalter einher [1]. Regelmäßiges Training schützt vor späterem Übergewicht, Hochdruck, Hyperlipoproteinämie und Herz-Kreislauf-Erkrankungen $[2,3]$, Darmkrebs und nicht insulinpflichtigem Diabetes [4]. Es erhöht den Mineralgehalt der Knochen und senkt das Risiko der Osteoporose und der in Folge eintretenden Frakturen [5]. Sport kann auch bei Asthma oder bei Mukoviszidose die Lebensqualität und die Belastbarkeit des Kindes erhöhen [6, 7]. Hinzu kommen die indirekten Vorteile des Sports. Er steigert das Selbstbewußtsein und Wohlbefinden und soll negativ mit Rauchen und Alkohol korrelieren $[8,9]$. Sport fördert auch die moralische Entwicklung des Kindes und erzieht zu Fairneß ${ }^{\prime}$ und sozialem Verhalten [10]. Zu möglichen Überbeanspruchungen insbesondere durch Leistungssport siehe Huber [11].
Der natürliche Bewegungsdrang des Kindes wird allerdings im Verlauf seiner Sozialisation eingeschränkt. Schon im Kindergarten nimmt der Anteil der Beschäftigungen im Sitzen zu. In der Schule ist es mit spontaner Bewegung, mit Ausnahme der Turnstunden, vorbei, obwohl es immer wieder Ansärze (Bewegte Schule) gibt, die Stunden mit Bewegungsübungen aufzulockern. Bewegung und körperliche Aktivität sind der Freizeit vorbehalten. $\mathrm{Da}$ aber in unserer Gesellschaft die Kindheit aus der natürlichen Umwelt stärker in die Wohnung verlegt wird, schon der Gefahren wegen, die dem Kind drohen (Verkehr, Gewalt, Drogendealer, sexuelle Übergriffe), wird es schwieriger, Sport zu treiben. Die Beschränkung auf geschlossene Räume leistet passiven Haltungen wie dem Fernsehen Vorschub.

Deswegen nimmt besonders mit der Einschulung die körperliche Aktivität ab. Dieser Trend setzt sich in der Pubertät fort. Adoleszenten kehren sich entwicklungsbedingt von vielen Attitüden der Erwachsenenwelt ab, ein Teil aber übernimmt die sitzende Lebensweise der Erwachsenen: Erkundung von der Couch. Deswegen mahnen z. B. Kelder et al. [12], die Erziehung zu körperlicher
Aktivität vor dem zwölften Lebensjahr zu beginnen, ehe sich Einstellungen verfestigen.

Es sind amerikanische Autoren, die den körperlichen Zustand ihrer Schulkinder im Vergleich zu europäischen Kindern beklagen. DiNubile [13] gibt an, daß von den Teens in Amerika $50 \%$ aller Mädchen und $30 \%$ aller Jungen die Meile nicht in zehn Minuten laufen können. Dietz/Gortmaker [14] schreiben, daß ein Schulkind in der Woche 20 bis 30 Stunden vor dem Fernseher verbringt. Kuntzleman bestätigt und ergänzt diese Einschätzung, wenn er sagt, daß nicht nur in mehreren Staaten der USA gar kein Sportunterricht betrieben wird, sondern daß der Sportunterricht selbst völlig ungenügend ist und nur wenige Minuten Aktivität verlangt - die übrige Zeit vergeht mit Vorbereitung und Warten. Hinzu kommt, daß die Eltern die Neigung der Kinder fernzusehen begünstigen - als den wichtigsten Babysitter der Nation [15]. Vermutet wird, daß eine Folge des Rückgangs körperlicher Aktivität bei unveränderter Ernährung das verbreitete Übergewicht ist [16].

Seit 1990 konzentriert man sich in den USA auf schulische Interventionen, die 
einen guten Sportunterricht und qualitativ bessere Ernährung sichern sollen, wie Heart Smart [17], die stark publizierte CATCH-Studie [18] oder die Bogalusa Herzstudie [19]. 1993 wurden aus dem MHHP (Minnesota Heart Hcalth Programm) bercits erste Interventionsergebnisse gemeldet: danach sollen insbesondere bei Mädchen signifikante Verbesserungen erzielt worden sein [20].

Vergleichbare deutsche Daten sind rar, doch sind die Ergebnisse weniger beunruhigend. In Deutschland liegen die Shell-Studie aus dem Jahr 1985 und die Ergebnisse der GIK-I-Studie vor, die 1991 veröffentlicht wurde. Die ShellStudie [21] stellte fest, daß unter den 14-17jährigen $77 \%$ Sport trieben. Nur $16 \%$ der Jungen und $28 \%$ der Mädchen waren inaktiv. In der GIK-I-Studie fanden die Autoren nur $2 \%$ Probanden, die keine körperliche Akrivitär angaben [22]. Im Rahmen des Forschungsschwerpunktes Epidemiologie und Prävention des Forschungsvorhabens "Herz-Kreislauf-Krankheiten« der DDR wurden mehrere Untersuchungen an Jugendlichen durchgeführt. Die Zahlen der Sportaktiven sind dort in der gleichen Altersstufe noch höher gewesen, sie lagen durchweg bei $90 \%$ und darüber; bei Mädchen um $80 \%$ in den einzelnen Klassenstufen [23]. In einer kleineren Untersuchung im Breisgau 1992 wurden die Freizeitvorlieben von 389 Jugendlichen insgesamt erhoben. Dabei lag in einer Rangreihe der Vorlieben Sport nach »Musik hören $«(82 \%)$ und seinfach mit anderen zusammensein $\approx(70 \%)$ mit $64 \%$ an dritter Stelle und schlug das Fernschen um einen Punkt [24].

\section{Material und Methode}

Die GIK-I-Studie untersuchte 1983/85 sowohl das Sportverhalten als auch die körperliche Aktivität in Berlin und Bremen. In der Wiederholungsuntersuchung 1994/95 (nur in Berlin) befragten wir rund 5000 Gymnasiasten und Hauptschüler repräsentativ für die Stadt. Um die soziale Struktur zu berücksichtigen, waren bereits in der GIK-I-Studie die Schulen nach der Sozialstruktur der Stadtbezirke in einer geschichteten Auswahl festgelegt worden. 1994/-95 wurden in West-Berlin die gleichen Schulen (mit geringen Veränderungen) untersucht, im hinzugekommenen Ost-Berlin wurde ebenfalls die Sozialstruktur der Bezirke berücksichtigt. Der Anteil der von uns untersuchten

Tabelle 1: Mittlere Intensitätswerte der von uns verwendeten Aktivitäten bzw. Aktivitätsgruppen und Saisonkorrekturen nach Fuchs [28]

\begin{tabular}{lcc}
\hline Sportart & MET & Korrekturfaktor \\
\hline Trimmen-Dauerlauf-Joggen & 9 & 9 \\
Ballspicle (z. B. Handball, Volleyball, & & \\
Basketball) & 7 & 12 \\
Rollschuh-Rollbrett (Skateboard)fahren & 7 & 6 \\
I Jockey-Eishockey & 8 & 3 \\
Schwimmen & 4 & 5 \\
Radfahren & 5 & 9 \\
Schlittschuhlaufen & 8 & 3 \\
Fußballspiclen & 12 & 9 \\
Turnen-Gymnastik-Ballett & 5 & 12 \\
Tanzen & 6 & 12 \\
Tischtennis & 4 & 9 \\
Sonstige Aktivität & 7 & 9 \\
\hline
\end{tabular}

Grundgesamtheit an der Schülergesamtheit beträgt $54 \%$. Die Hauptschüler machen $7 \%$, die Gymnasiasten $47 \%$ aller Schüler aus. Die übrigen Schüler verteilen sich auf Real-, Gesamt- und Berufsschulen, die hier nicht untersucht wurden; ihr Verhalten liegt zwischen den von uns befragten Gruppen.

Vergleiche mit der GIK-I-Studie der Jahre 1983/85 werden nur mit deutschen Kindern aus West-Berlin angestellt, um Verzerrungen auszuschließen.

Zur Erfassung körperlicher Aktivitäten ist die genaueste Methode die Beobachtung oder das Protokoll. Aus Gründen der Forschungsökonomie wird jedoch meist die Befragung gewählt, das einzige Instrument, um Daten in größeren Stichproben zu sammeln. Am häufigsten sind die auch in den GIK-Studien verwandten relativ groben Fragen zum Ausmaß des Sporttreibens. Seit einiger Zeit versucht man, aktive von inaktiven Probanden durch die Schätzung übungsinduzierten Schwitzens zu trennen $[25,26]$. Es werden jedoch inzwischen auch detailliertere Erhebungen verwandt, die zugleich das Ausmaß des Körperstoffwechsels infolge körperlicher Aktivität abzuschätzen versuchen. Fin Vergleich von acht dieser Fragebogen in den USA hat gezeigt, daß verschiedene Erhebungsinstrumente zu nicht kompatiblen Ergebnissen führen [27]. Es ist wichtig, insbesondere bei Wiederholungsuntersuchungen, mit den gleichen Instrumentarien $z u$ arbeiten.

In unserem Fall übernahmen wir den Frhebungsbogen der Berlin-BremenStudie, worin die verschiedenen Formen sportlicher Aktivität als Produkt aus
Häufigkeit und Dauer erfaßt werden. Dabei wird mit einem Saisonfaktor korrigiert, und die zwölf Aktivitätsformen werden in ihrer Stoffwechselintensität eingeschätzt. Insgesamt liegen am Ende für jedes Kind drei Maßzahlen vor: Die Sportstundenzahl pro Monat insgesamt, eine Sportstundenzahl pro Monat für hoch und für niedrig belastende Aktivitätsarten. Diese Intensität wird als Verhältniszahl zwischen der Energieverausgabung während der sportlichen Aktivität zum Energicumsatz im Ruhezustand (Grundumsatz) aufgefaßt. Sie wird in der Regel in MET ausgedrückt (metabolic unit). Da sie bei einer Befragung nicht direkt erhoben werden kann, hat Fuchs [28] in Anlehnung an andere [29-31] eine Tabelle durchschnittlicher Intensitätswerte aufgestellt, die auch wir verwenden (Tab. 1).

Bei detaillierten Fragen nach einzelnen Aktivitäten nehmen die Schwierigkeiten für den Probanden zu, korrekt zu antworten. Die Antwort hängt vom Erinnerungsvermögen und der Fähigkeit ab, die Gesamtheit der Antworten zu koordinieren. In der Messung durch die Vorgabe von zwölf populären Sportarten kam es zu Überschätzungen, vor allem durch Schüler der 7. Klasse. So hat ein Schüler fast alle Sportarten mit maximaler Dauer und Häufigkeit gewählt und kam auf 736 Stunden. Ein Monat von 30 Tagen zählt 720 Stunden. Der Anteil dieser Überschätzer insgesamt ist zwar gering $(0,5 \%>300 \mathrm{~h})$, doch haben wir für Mittelwertberechnungen die höchstmögliche Stundenzahl auf $300 \mathrm{~h}$ begrenzt.

\section{Unsere Hypothesen sind:}

1. Nach wie vor treiben Jungen mehr Sport als Mädchen. 
Tabelle 2: Treibst Du gerne Sport? West-Berliner Schüler der 8.-10. Klasse in Hauptschulen und Gymnasien (ohne ausländische Mitschüler) 1994/95 (7. Klassen nicht vergleichbar befragt; drei Vorgaben ausgewertet, Angaben in \%)

\begin{tabular}{lcccc}
\hline & \multicolumn{2}{c}{ Hauptschule } & \multicolumn{2}{c}{ Gymnasium } \\
& Jungen & Mädchen & Jungen & Mädchen \\
\hline 1983/85: Ja, sehr & 47 & 38 & 65 & 51 \\
1994/95: Ja, sehr & 51 & 31 & 58 & 41 \\
1983/85: Nein, ziemlich & & & & 3 \\
wenig bzw. gar nicht & 5 & 11 & 0 & 10 \\
1994/95: Nein, ziemlich & & & & \\
wenig bzw. gar nicht & 9 & 18 & & \\
& & & & 1,7 \\
Mittelwerte der 5stuf. Skala & & & 1,5 & 1,9 \\
1983/85: & 1,9 & 2,2 & 1,6 & \\
1994/95: & 1,7 & 2,2 & & \\
\hline
\end{tabular}

Tabelle 3: Machst du in deiner Freizeit ein regelmäßiges Trainingsprogramm? West-Berliner Schüler der 7.-10 Klasse in Hauptschulen und Gymnasien (ohne ausländische Mitschüler) im Vergleich 1983/85 und 1994/95

\begin{tabular}{lcccc}
\hline & \multicolumn{2}{c}{ Hauptschule } & \multicolumn{2}{c}{ Gymnasium } \\
& Jungen & Mädchen & Jungen & Mädchen \\
\hline $\mathrm{Ja}(1983 / 85)$ & 44 & 30 & 60 & 49 \\
$\mathrm{Ja}(1994 / 95)$ & 44 & 22 & 55 & 45 \\
\hline
\end{tabular}

Tabelle 4: Wie oft machst du in deiner Freizeit so Sport, daß du dabei außer Puste kommst oder ins Schwitzen gerätst? West-Berliner Schüler der 7.-10. Klasse in Hauptschulen und Gymnasien (ohne ausländische Mitschüler) im Vergleich 1983/85 und 1994/95

\begin{tabular}{lcccc}
\hline & \multicolumn{2}{c}{ Hauptschule } & \multicolumn{2}{c}{ Gymnasium } \\
& Jungen & Mädchen & Jungen & Mädchen \\
\hline 1983/85: Täglich oder & & & & \\
mehrmals wöchentlich & 62 & 25 & 62 & 44 \\
1994/95: Täglich oder & 57 & 27 & & \\
mehrmals wöchentlich & 19 & 33 & 64 & 41 \\
1983/85: seltener als einmal & & & 8 & 10 \\
im Monat oder nie & 18 & 35 & & \\
1994/95: seltener als einmal & & & 8 & 20 \\
im Monat oder nie & 3 & 4,1 & & \\
Mittelwerte der 7stuf. Skala & 3,1 & 4,1 & 2,7 & 3 \\
1983/85: & & & 2,6 & 3,3 \\
1994/95: & & &
\end{tabular}

2. Sport als trainierte Leistung wird stärker unter Gymnasialschülern betrieben. Sportliche Aktivität insgesamt ist jedoch unter Hauptschülern verbreiteter als unter Gymnasiasten, weil diese stärker außerschulisch belastet sind.

3. Das Sporttreiben nimmt mit zunchmendem Lebensalter ab, weil neue Interessen mehr Zeit brauchen.

4. Insgesamt hat das Sporttreiben gegenüber der ersten Untersuchung vor zwölf Jahren abgenommen.
Gymnasialschüler geben eine höhere Sportvorliebe an als Hauptschüler. Dies trifft unverändert für beide Erhcbungsphasen zu (Tab. 2).

Auch hier sind die Jungen den Mädchen in der jeweiligen Schulform voraus, Schüler und Schülerinnen aus Gymnasien sind häufiger dabei, regelmäßig zu trainieren als solche aus Hauptschulen. Es scheint, daß besonders unter Mädchen, aber auch unter Jungen aus Gymnasialklassen, die Teilnahme am regelmäßigen Training zurückgegangen ist (Tab. 3).

Ein guter Indikator für intensives Training ist die in unserem Fragebogen enthaltene Frage, ob man während der Übungen schwitzt bzw. außer Puste gerät. Nur wenn der Kreislauf die Ruhepulsfrequenz um mehr als etwa zwei Drittel (altersabhängig) übersteigt, wird überhaupt ein Trainingseffekt erzielt. Diese Belastung sollte wenigstens drei$\mathrm{mal}$ in der Woche mindestens $30 \mathrm{Minu}-$ ten lang stattfinden (eine der letzten Veröffentlichungen dazu stammt von Pate et al. [32]). Unsere Frage unterscheidet grob zwischen effektivem Training und körperlicher Aktivität, die mehr der Kommunikation allein dient (Tab. 4). Auch hier sind es mehr Jungen als Mädchen, die mit physiologischem Effekt trainieren.

In dieser Tabelle zeigt sich, daß Gymnasialschülerinnen den Sport ernster nehmen und stärker trainieren als die gleichaltrigen Schülerinnen der Hauptschule. In der Kondition und im Aktivitätsumfang (Tab. 5, 6, Abb. 1, 2) liegen Jungen in allen Schulformen deutlich vorn. Die körperliche Kondition hängt nicht allein von der Aktivität des Jugendlichen $a b$, sondern auch von genetischen und psychischen Faktoren. Man darf zudem nicht vergessen, daß die Frage das Prestige berührt. Mädchen geben leichter körpcrliche Beeinträchtigungen zu als Jungen. Diese schätzen sich als besser konditioniert ein als Mädchen, Gymnasialschüler besser als Hauptschüler.

Im körperlichen Aktivitätsumfang, der sich aus der Summe der Angaben der verschiedenen sportlichen Betätigungen ergibt, liegen cbenfalls die Jungen vorn. Sie geben durchweg eine höherc Stundenzahl pro Monat an als Mädchen. Der Unterschied ist hochsignifikant. Ebenfalls signifikant $(p=0,001)$ ist der Unterschied zwischen Hauptschülern und Gymnasiasten. Für die allgemeinen körperlichen Aktivitäten verwenden 
Hauptschüler viel mehr Zcit als die Schülerinnen und Schüler der Gymnasien.

Eindeutig fällt ebenso der erwartete Rückgang innerhalb der einzelnen Klassenstufen aus, ein Fakt, den schon die Untersucher 1983 feststellten, als sie im Längsschnitt und damit noch präziser untersuchten (Abb. 3 und 4). Zwar ist der Rückgang von Stufe zu Stufe ungleichmäßig, doch liegen signifikante Unterschiede zwischen der 7. und 10. Klasse vor. Ähnliche Ergebnisse sind z. B. aus Norwegen und der Schweiz bekannt $[33,34]$.

In der vierten Hypothese nahmen wir an, daß die deutschen Kinder und Jugendlichen ähnlich wie die Amerikaner einen Rückgang bei der körperlichen Aktivität aufzuweisen haben, weil vieles für eine Zunahme der sitzenden Lebensweise auch in Deutschland spricht. Einem leichten Rückgang bei Gymnasialschülern steht ein Anstieg bei Hauptschülern gegenüber. Innerhalb der Aktivitäten gibt es bei Jungen eine Verschiebung zu höher beanspruchenden Sportarten, der auch zu einer Zunahme der Aktivität bei Gymnasiasten führt. Die Hypothese des Rückgangs insgesamt (für alle Schüler) kann mit uns̄eren Ziffern nicht beantwortet werden, weil es sowohl Rückgang als auch Anstieg gibt und die Sportzahlen der Gesamt-, Realund Berufsschüler nicht erfaßt sind. Einen Rückgang scheint es in der sportlichen Organisiertheit zu geben (Tab. 7).

Die unter der heutigen Schülergeneration beliebtesten körperlichen Aktivitäten, gemessen an der auf sie verwendeten Gesamtstundenzahl, gibt Tabelle 8 an.

\section{Determinanten körperlicher Aktivität}

Schon in den Vorstudien zur GIK-IStudie 1983/85 wurde nachgewiesen, $\mathrm{daß}$ die größte Bedeutung für das Sportverhalten des Jugendlichen dem Einfluß der »important others« zukommt, den ihm wichtigen Personen des sozialen Umfeldes. In erster Linie sind hier dic gleichaltrigen Freunde, die Clique zu nennen, der beste F'reund und schließlich die Eltern. Von Einfluß sind auch die eigenen Überzeugungen und der Spaß an der Sache [35-37]. Craig et al. [38] fanden signifikante Zusammenhänge mit der eigenen Absicht, Sport zu treiben, und mit der Zuversicht, dies auch zu können (perceived behavioral control, einem zentralen Begriff der
Tabelle 5: Wie ist deine körperliche Kondition? Berliner Schüler der 7.-10. Klasse in Hauptschulen und Gymnasien (ohne ausländische Mitschüler) im Vergleich 1983/85 und 1994/95

\begin{tabular}{lcccc}
\hline & \multicolumn{2}{c}{ Hauptschule } & \multicolumn{2}{c}{ Gymnasium } \\
& Jungen & Mädchen & Jungen & Mädchen \\
\hline 1983/85: & 50 & 37 & & \\
sehr gut, gut & 40 & 52 & 62 & 45 \\
mittel & 10 & 11 & 33 & 43 \\
nicht besonders, gar nicht gut & 2,4 & 2,7 & 5 & 12 \\
Mittelwert & & & 2,2 & 2,6 \\
1994/95: & 59 & 39 & & \\
sehr gut, gut & 31 & 46 & 61 & 51 \\
mittel & 10 & 15 & 28 & 38 \\
nicht besonders, gar nicht gut & 2,3 & 2,7 & 10 & 11 \\
Mittelwcrt & & & 2,3 & 2,5 \\
\hline
\end{tabular}

Tabelle 6: Index der körperlichen Aktivität unter West-Berliner Schülern (8.-10. Klasse) in Stunden pro Monat 1983/85 und 1994/95 (saisonkorrigierte und anteilgewichtete Mittelwerte, ohne ausländische Schüler und ohne 7. Klasse, da 1983 mit anderem Wortlaut befragt)

\begin{tabular}{lcccccc}
\hline & \multicolumn{2}{c}{ Gesamtaktivität } & \multicolumn{2}{c}{ davon: hochbelastet } & \multicolumn{2}{c}{ niedrig belastet } \\
& männl. & weibl. & männl. & weibl. & männl. & wcibl. \\
\hline Hauptschule 83 & 74 & 47 & 32 & 15 & 42 & 32 \\
Hauptschule 95 & 78 & 56 & 39 & 18 & 39 & 38 \\
Gymnasium 83 & 56 & 39 & 28 & 14 & 28 & 25 \\
Gymnasium 95 & 55 & 34 & 31 & 12 & 24 & 22 \\
\hline
\end{tabular}
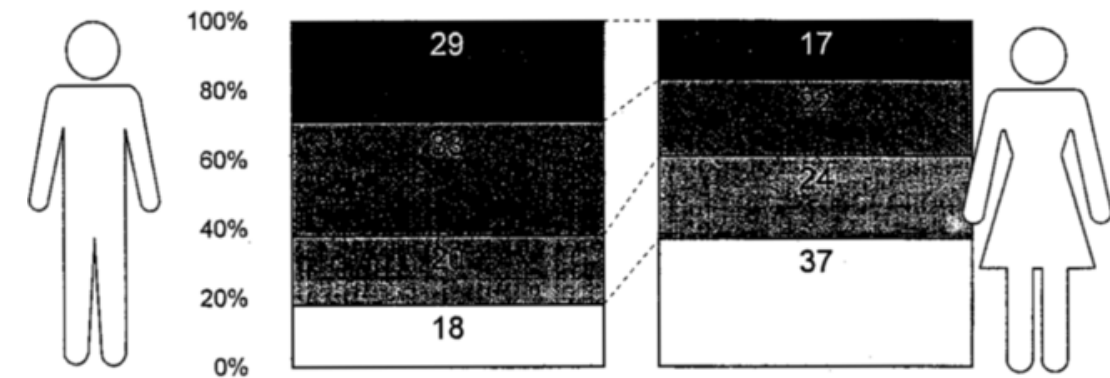

$$
<25 \mathrm{~h} / \text { Monat } 25-<50 \mathrm{~h} 50-<100 \mathrm{~h}=100 \mathrm{u} . \mathrm{mehr}
$$

Abbildung 1: Hauptschüler/innen - Anteile körperlicher Aktivität nach Geschlecht.
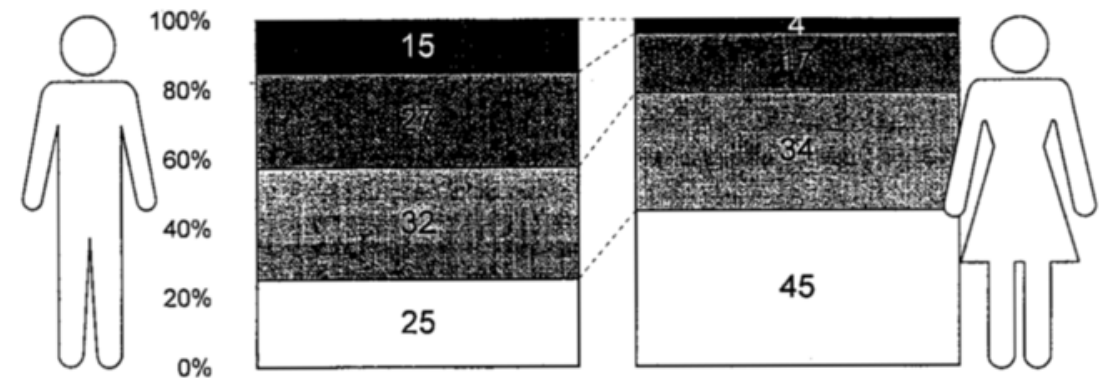

$<25 \mathrm{~h} /$ Monat $\square 25-<50 \mathrm{~h}$ 四 $50-<100 \mathrm{~h} \square 100 \mathrm{u} . \mathrm{mehr}$

Abbildung 2: Gymnasialschüler/innen - Anteile körperlicher Aktivität nach Geschlecht. 

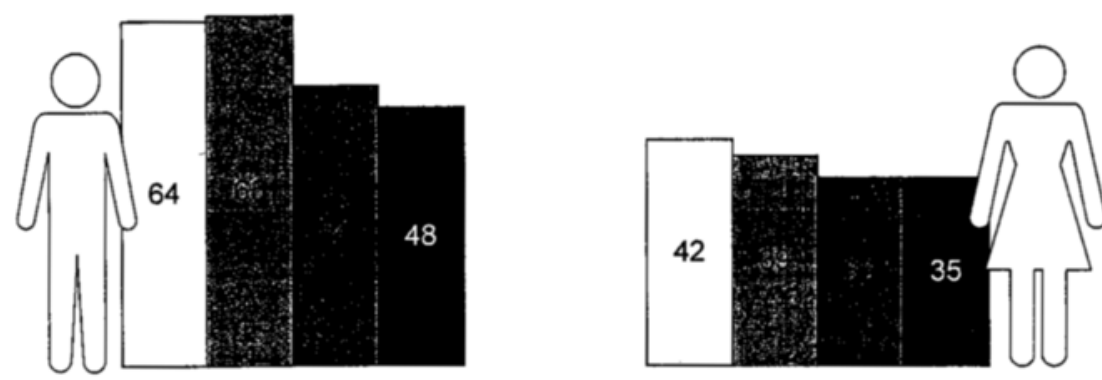

\section{Klasse 8 . Klasse $\mathbf{9}$ 9. Klasse $\mathbf{1 0 .}$ Klasse}

Abbildung 3: Gymnasialschüler/innen - Körperliche Aktivität in h/Monat nach Geschlecht und Klassenstufen.
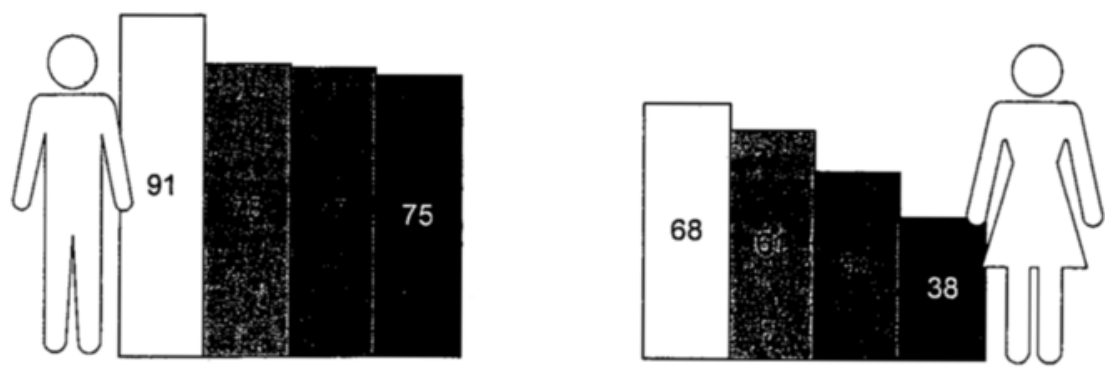

\section{Klasse}

8. Klasse

9. Klasse

10. Klasse

Abbildung 4: Hauptschüler/innen - Körperliche Aktivität in h/Monat nach Geschlecht und Klassenstufen.

Lerntheorie von Bandura). Dieser Faktor der Verhaltenskontrolle wicderum war signifikant abhängig von der Überzeugung, in Sport gut zu sein. Auch die Ethnizität spielte eine Rolle (Weiße trieben mehr Sport), der BMI korrelierte negativ mit sportlicher Aktivität. Reynolds et al. [39] nennen bei 16jährigen signifikante Zusammenhänge mit dem eigenen Willen, aktiv zu sein (um den eigenen Körper in einen guten Zustand zu bringen), und für die Kategorie Selbstbewußtsein (self-efficacy). Biddle hat in einer Metaanalyse [40] gezeigt, daß von den psychosozialen Variablen in den gegenwärtigen Untersuchungen zum Sportreiben vor allem Verbesserung von Depressivität und ein höheres Selbstbewußtsein infolge Sport genannt werden.

In allen Untersuchungen sind natürlich Geschlecht und Alter wichtige Größen.

Wir haben an unseren Daten aus den Jahren 1994/95 die wichtigsten hier genannten Einflußfaktoren in einer logistischen Regression überprüft und zwar:

Alter, Geschlecht, Schultyp, Wohnsitz in Ost- bzw. West-Berlin, sportlich organisiert, Sportverhalten des Vaters, Sportverhalten der Mutter, der Geschwister, des besten Freundes, Meinung der Freunde zum Sport insgesamt, Selbstbewußtsein, negatives Befinden (Depressivität bzw. Ängstlichkeit), Beliebtheit, Copingfähigkeit und gesundheitsbezogene Kontrollüberzeugungen. Die stetige Variable "Sportliche Akti-

Tabelle 7: Bist du Mitglied in einem Sportverein? West-Berliner Schüler der 7.-10. Klasse in Hauptschulen und Gymnasien (ohne ausländische Mitschüler) im Vergleich 1983/85 und 1994/95

\begin{tabular}{llccc}
\hline & \multicolumn{2}{c}{ Hauptschule } & \multicolumn{2}{c}{ Gymnasium } \\
& Jungen & Mädchen & Jungen & Mädchen \\
\hline 1983/85: ja & 39 & 25 & 64 & 56 \\
1994/95: ja & 35 & 19 & 55 & 40 \\
\hline
\end{tabular}

vität « wurde dafür dichotomisiert (Mittelwert).

In der Tabelle 9 lautet die Zielvariable »Algemeine sportliche Aktivität «, in 10 »Hoch beanspruchende sportliche Aktivität «, in 11 und 12 »Sportliche Intensität «, wobei in 12 nur die Population derer einbezogen wurde, die hoch beanspruchende Aktivitäten betreiben. Bei den Einflußfaktoren zeigt sich in der Aufeinanderfolge der Tabellen, daß zunächst der Schultyp, dann das Geschlecht und schließlich auch die Teilnahme von Freunden an Bedeutung verlieren. Stärkste Faktoren sind am Ende, bei jenen, die intensiv und viel Sporttreiben, der eigene Spaß am Sport, das Selbstbewußtsein und die Tatsache, daß man sportlich organisiert ist. Zugleich ändert sich der Schultyp. Am Anfang, unter der Zielvariablen "Allgemeine sportliche Aktivität«, lagen die Hauptschüler weit vorn. Jetzt, bei den hoch Motivierten, sind es die Gymnasiasten, die stärker Sport treiben und zwar Jungen und Mädchen gleichermaßen. Die kleineren Zahlen verringern die Signifikanz der Unterschiede.

Dic Zielvariable "Sportliche Aktivität" der Tabelle 9, die sowohl wenig als stark belastende Sportarten umfaßt, nennt als Haupteinflußgröße den Schultyp. Hauptschüler sind stärker in allgemeiner sportlicher Akrivität involviert, Jungen mehr als Mädchen. Spaß am Sport ist ein starker Antrieb, ebenso das Engagement in Sportvereinen, die in Deutschland sehr verbreitet sind. Signifikant sind auch die Einflüsse der engeren Gruppe bzw. des besten Freundes. Elterneinfluß ist dagegen nur gering vorhanden. Der Ost-West-Unterschied beruht auf höheren Aktivitätszahlen der Ost-Berliner Schüler.

Wenn man die Zielvariable ändert und die Variablen untersucht, die den Vektor "Hoch belastende Aktivität « laden (Tab. 10), so zeigt sich noch immer etwa die gleiche Reihenfolge; zusätzlich tritt mit dem Einfluß des Vaters ein signifikanter Einfluß des Elternhauses hinzu. Doch sind die Odds Ratios ausgewogener. Der Schultyp verliert gegenüber dem Geschlecht an Einfluß, sportliche Organisierthcit wie auch der Einfluß des Freundes wirken stärker.

In der Tabelle 11 wurde die Zielvariable abermals verändert. Es wird jetzt nach der Risikowahrscheinlichkeit einzelner Variablen im Zusammenhang mit der Variable »Sportliche Intensität « gefragt. Bedingung dafür ist, daß der Schüler 
oder die Schülerin mehrmals in der Woche oder täglich beim Freizeitsport außer Atem gerät bzw. schwitzt - ein einfaches Merkmal für ein effektives Training. Es zeigt sich, daß jetzt der Sport zum Selbstzweck wird: Der Hauptantrieb ist Spaß. Der Einfluß des Schultyps verliert sich, dagegen nimmt die Rate sportlicher Organisiertheit zu. Wahrscheinlich ist die Mehrheit dieser Jugendlichen in Sportvereinen. Die Bedeutung des Geschlechtes nimmt ab, doch sind Jungen noch immer stärker vertreten. Ein neuer Faktor kommt hinzu: Selbstbewußtsein, eine Eigenschaft, die vonnöten ist, um solche Trainingsintensität durchzuhalten, aber zugleich auch ein Ergebnis sportlicher Aktivität darstellt.

In der Tabelle 12 ist die Zielvariable der Regressionsgleichung nach wie vor dic "Sportliche Intensität", jedoch ist die Stichprobe auf jene Schüler beschränkt worden, die eine hohe körperliche Belastung beim Sporttreiben genannt haben.

Das Geschlecht ist jetzt als Einflußgröße verschwunden, der Schultyp wieder hinzugetreten (der kleineren Zahl wegen nicht signifikant), hat aber seine Richtung gewechselt: In dieser mit hoher Intensität trainierenden Gruppe sind stärker Schüler bzw. Schülerinnen des Gymnasiums zu finden. Sportliche Organisiertheit und Spaß am Sport sind nach wie vor dominierende Merkmale.

\section{Diskussion}

Unsere Ergebnisse stützen und variieren die Mitteilungen aus anderen Studien. Auf den bloßen Umfang körperlicher Aktivitäten haben die Variablen Hauptschule, männliches Geschlecht und Freunde den größten Einflußß. Je intensiver Sport betrieben wird, um so stärker wird die eigene Motivation, der Spaß an der Sache und die Organisiertheit. Gymnasiasten sind in der letzten Gruppe stärker beteiligt als Hauptschüler, unabhängig vom Geschlecht. Möglicherweise wird damit auch ein Widerspruch geklärt, der in einigen Untersuchungen Gymnasiasten, in anderen Hauptschüler als besonders aktiv darstellt, etwa [41].

Ganz ähnliche Ergebnisse zeigen sich, wenn man die Schüler der ersten Welle 1983/85 untersucht. Sowohl beim Stundenumfang als auch der Intensität des Sporttreibens liegen die Jungen vorn $(\mathrm{OR}=2,1$ bzw. 2,5). Ebenso motivierend sind Mitgliedschaft im Sportverein
Tabelle 8: Rangfolge der ausgeübten Sportarten unter Berliner Schulkindern der 7.-10. Klasse in Hauptschulen und Gymnasien nach Umfang und Geschlecht (Deutsche und Ausländer 1994/95)

\begin{tabular}{|c|c|c|c|c|c|}
\hline mänr & lich & & weib] & & \\
\hline Sportarten & h/Mon & $\mathrm{TR}^{\prime}$ & Sportarten & $\mathrm{h} / \mathrm{Mon}$ & TR \\
\hline 1. Rad fahren & 44433 & $90 \%$ & 1. Schwimmen & 22620 & $94 \%$ \\
\hline 2. Fußball & 44339 & $78 \%$ & 2. Tanzen & 20364 & $80 \%$ \\
\hline 3. Hand-, Volley-, & & & 3. Rad fahren & 17166 & $88 \%$ \\
\hline Basketball & 31544 & $85 \%$ & 4. Hand-, Volley-, & & \\
\hline 4. Schwimmen & 29230 & $94 \%$ & Baskerball & 12434 & $79 \%$ \\
\hline 5. Sonst. Sport ${ }^{2}$ & 13759 & $34 \%$ & 5. Gymnastik & & \\
\hline 6. Dauerlauf, Jogg. & 11710 & $73 \%$ & Ballett, Turnen & 10897 & $67 \%$ \\
\hline 7. Tischtennis & 11595 & $80 \%$ & 6. Sonst. Sport & 7186 & $28 \%$ \\
\hline 8. Tanzen & 8864 & $56 \%$ & 7. Tischtennis & 5330 & $76 \%$ \\
\hline 9. Gymnastik & & & 8. Dauerlauf, Jogg. & 4376 & $68 \%$ \\
\hline Ballett, Turnen & 4443 & $35 \%$ & 9. Schlittschuhe & 3893 & $81 \%$ \\
\hline 10. Schlittschuhe & 4096 & $59 \%$ & 10. Fußball & 3854 & $37 \%$ \\
\hline 11. Rollschuhe, & & & 11. Rollschuhe, & & \\
\hline Skateboard & 2891 & $36 \%$ & Skateboard & 2282 & $51 \%$ \\
\hline 12. (Eis)Hockey & 1019 & $23 \%$ & 12. (Eis)Hockey & 209 & $11 \%$ \\
\hline Insgesamt & 207923 & & & 110611 & \\
\hline
\end{tabular}

$1 \mathrm{TR}=$ Teilnahmerate

$2 \mathrm{Zu}$ den sonstigen Sportarten, die nicht in unserer Vorgabe enthalten waren, zählen bei Jungen Judo, Boxen und andere Kampfsportarten (290 Nennungen), Bodybuilding, Tennis und Wassersport; bei den Mädchen Reiten (207 Nennungen), ebenfalls Kampfsportarten und Tennis.

Tabelle 9: Einflußfaktoren auf die Zielvariable »Index der körperlichen Gesamtaktivität * unter Berliner Schülern (7.-10. Klasse) 1994/95 (ohne ausländische Schüler)

\begin{tabular}{lccc}
\hline Einflußfaktoren & Odds Ratios & Signifikanz & CI(95\%) \\
\hline Alter & 1,1 & 0,0321 & $1,0-1,2$ \\
Schultyp & 4,4 & 0,0000 & $3,2-5,9$ \\
Spaß am Sport & 3,1 & 0,0000 & $2,2-4,4$ \\
Sportlich organisiert & 1,8 & 0,0000 & $1,4-2,4$ \\
Geschlecht & 1,7 & 0,0001 & $1,4-2,2$ \\
beste(r) Freund(in) treibt Sport & 1,6 & 0,0021 & $1,2-2,1$ \\
Meinung der Freunde zum Sport & 1,5 & 0,0067 & $1,1-1,9$ \\
gesundheitsbez.ogene Kontrollüberzeugungen & 1,5 & 0,0043 & $1,1-1,0$ \\
Ost-/West-Berlin & 1,4 & 0,0096 & $1,1-1,8$ \\
Vater treibt Sport & 1,2 & 0,0950 & $0,9-1,7$ \\
Mutter treibt Sport & 1,2 & 0,2350 & $0,9-1,6$ \\
Geschwister treiben Sport (falls vorhanden) & 1,3 & 0,1030 & $0,9-1,5$ \\
Selbstbewußtsein & 1,3 & 0,0931 & $0,9-1,9$ \\
Copingfähigkeit & 1,6 & 0,0644 & $0,9-1,5$ \\
Beliebtheit & 1 & 0,7824 & $0,8-1,4$ \\
Depressivität I & 1,2 & 0,3218 & $0,8-1,6$ \\
\hline
\end{tabular}

Tabelle 10: Einflußfaktoren auf die Zielvariable »Index hoch belastender körperlicher Aktivität « unter Berliner Schülern (7.-10. Klasse) 1994/95 (ohne ausländische Schüler) (In den Tabellen 10-12 sind nur noch Variablen mit Signifikanzen < 0,01 dargestellt; es wurden alle in Tabelle 9 genannten Faktoren kontrolliert.)

\begin{tabular}{lccc}
\hline Einflußfaktoren & Odds Ratios & Signifikanz & CI \\
\hline Geschlecht & 3,3 & 0,0000 & $2,5-4,5$ \\
Schultyp & 2,8 & 0,0000 & $2,1-3,9$ \\
Spaß am Sport & 2,8 & 0,0000 & $1,9-4,2$ \\
Sportlich organisiert & 2,7 & 0,0000 & $2,1-3,7$ \\
beste(r) Freund(in) treibt Sport & 2,5 & 0,0000 & $1,7-3,6$ \\
Mcinung der Freunde zum Sport & 1,8 & 0,0002 & $1,3-2,4$ \\
Vater treibt Sport & 1,5 & 0,0078 & $1,1-2,1$ \\
\hline
\end{tabular}


Tabelle 11: Einflußfaktoren auf die Zielvariable $*$ Sportintensität « unter Berliner Schülern (7.-10. Klasse) 1994/95 (ohne ausländische Schüler)

\begin{tabular}{lccc}
\hline Einflußfaktoren & Odds Ratios & Signifikanz & CI \\
\hline Spaß am Sport & 4,2 & 0,0000 & $3,0-5,9$ \\
Sportlich organisiert & 3,7 & 0,0000 & $2,8-4,9$ \\
Geschlecht & 1,9 & 0,0000 & $1,5-2,5$ \\
Selbstbewußtsein & 1,7 & 0,0022 & $1,2-2,4$ \\
\hline
\end{tabular}

Tabelle 12: Einflußfaktoren auf die Zielvariable $»$ Sportintensität « unter Berliner Schülern mit hoch beanspruchender körperlicher Aktivität (7.-10. Klasse) 1994/95 (ohne ausländische Schüler)

\begin{tabular}{lccc}
\hline Einflußfaktoren & Odds Ratios & Signifikanz & CI \\
\hline Sportlich organisiert & 4,7 & 0,0000 & $7,7-8,4$ \\
Spaß am Sport & 2,9 & 0,0080 & $1,3-6,5$ \\
Selbstbewußtsein & 2,4 & 0,0191 & $1,2-4,9$ \\
Schultyp & 0,7 & 0,2446 & $0,4-1,3$ \\
\hline
\end{tabular}

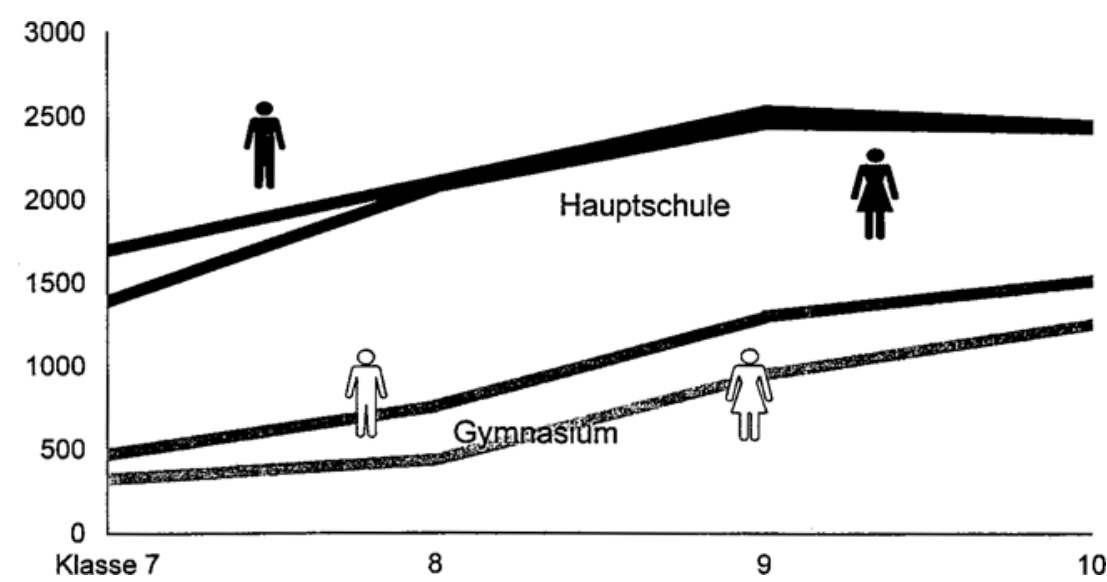

Abbildung 5: Zigarettenkonsum Berliner Schüler nach Klassenstufe, Schulform und Geschlecht in Stück/Jahr (ohne Ausländer).

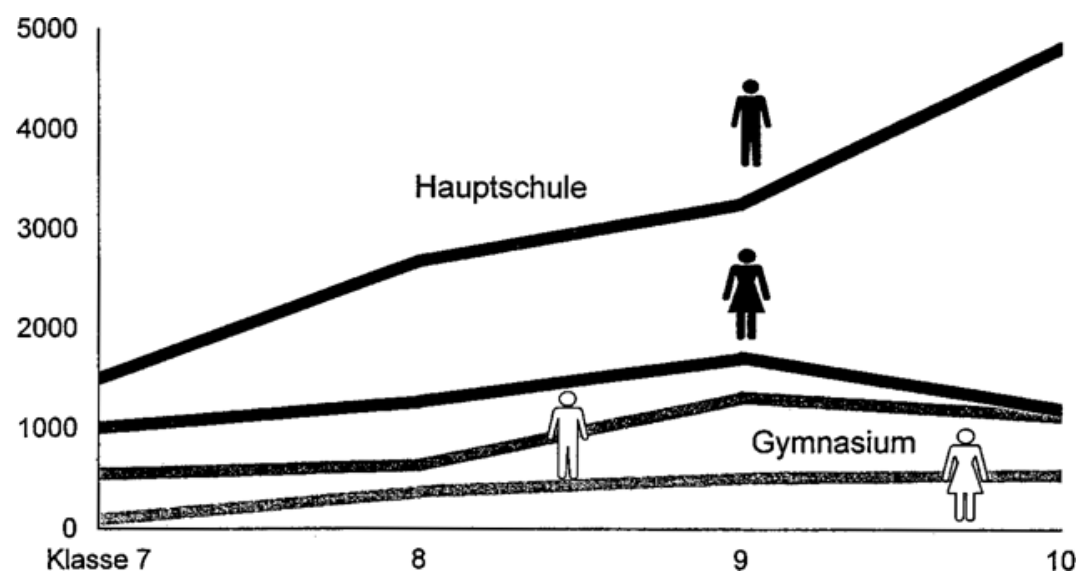

Abbildung 6: Alkoholkonsum Berliner Schüler nach Klassenstufe, Schulform und Geschlecht in g/Jahr (ohne Ausländer).
(OR=2,1 bzw. 2,3) und Freude am Sport ( $\mathrm{OR}=3,2$ bzw. 2,4). Etwas weniger zählt die Meinung der Freunde zum Sporttreiben $(O R=1,5$ bzw. 1,3). Bei der Intensität des Sporttreibens ergibt sich jedoch der gleiche Unterschied wie in der GIK-II-Studie. Bei der Stundenzahl führen die Hauptschüler $(O R=2,9)$, bei der Intensität geraten die Gymnasiasten stärker in den Blick (OR=0,8 ns).

Wir fanden in unserer Stichprobe im Gegensatz zu Röthlisberger/Calmonte [34] keine signifikanten Negativkorrelationen von körperlicher Aktivität mit Rauchen und Alkoholeinnahme. Sporttreiben geht zwar leicht mit geringerem Alkoholkonsum bzw. Zigarettenverbrauch einher, doch weit von der Signifikanzgrenze entfernt. Die körperliche Anstrengung nimmt $z$ wischen der 7. und 10. Klasse ab, während Alkoholgebrauch und Rauchen zunehmen (Abb. 5 und 6). Das betrifft Sportler und Nichtsportler gleichermaßen.

Die Schweizer erwähnten u.a., daß Sport die Gesundheits- und soziale $\mathrm{Zu}$ friedenheit erhöht. Wir haben in der GIK-II-Studie ebenfalls gesehen, daß die Gesundheitszufriedenheit und auch das Selbstbewußtsein unter Personen, die mehr Sport als der Durchschnitt angeben, deutlich höher sind: Sehr gesund und ziemlich gesund fühlen sich $91 \%$ der Jungen und $85 \%$ der Mädchen, die engagiert Sport treiben, gegenüber $67 \%$ der Jungen und $70 \%$ der Mädchen, die in geringerem Maße aktiv sind ( $p=0,0001$ ).

Dies könnte natürlich zur Ursache haben, daß nur gesunde Personen Sport treiben. Da die Mittelwerte auch der Nichtsporttreibenden jedoch im oberen Bereich zwischen der Gesundheitseinschätzung "sehr gut « und "ziemlich gut « angesiedelt sind, gehen wir davon aus, daß der Sport die Ursache für das Wohlbefinden ist. Jeder, der Sport treibt, kennt diesen Rückkopplungseffekt.

\section{Literatur:}

[1] Kaplan, G. A., Strawbridge, W. J., Cohen, R. D., and Hurgerford, L. R.: Natural history of leisuretime physical activity and its correlates: Association with mortality from all causes and cardiovascular disease over 28 years. Amer. J. Epidemiol. 144 (1996) 793.

[2] Francis, K.: Physical activity in the prevention of cardiovascular disease. Phys. Theraphy 76 (1996) 456 .

[3] Berg, A., Halle, M., Bauer, S., Korsten-Reck, U.,und Keul, J.: Körperliche Aktivität und Essverhalten: Strategien zur Verbesserung des Serumlipidprofils bei Kindern und Jugendlichen. Wien. Med. Wschr. 144 (1994) 138. 


\section{Originalien und Übersichtsarbeiten}

[4] Powell, K. E., Caspersen, C. J., Koplan, J. P., and Ford, E. S.: Physical activity and chronic diseases. Amer. J. Clin. Nutr. 49 (1989) 999.

[5] Cummings, S. R., Kelsey, J. L., Nevitt, M. C., and ODowd, K. J.: Epidemiology of osteoporosis and osteoporotic fractures. Epidemiol. Rev. 7 (1985) 178

[6] Hollmann, W.: Über Übung, Training und Sport beim asthmakranken Kind aus sportmedizinischer Sicht. Monatschr. Kinderheilkunde 133 (1985) 863.

r7] Eichinger, T., und Zimmermann, T.: Laufbandergometrie mit submaximaler Belastung bei Kindern und Jugendlichen mit Mukoviszidose (CF). Pncumologie 4 (1991) 906.

[8] Raitakari, O. T., Lcino, M., Räikkonen, K., Porkka, K. V. K., Taimela, S., Räsänen, L., and Viikari, J. S. A.: Clustering of risk habits in young adults. Amer. J. Epidemiol. 142 (1990) 36.

[9] Thorlindsson, T., Vilhjalmsson, R., and Valgeirsson, G.: Sport participation and perceived health status: a study of adolescents. Soc. Sci. Med. 31 (1990) 551.

[10] Gibbons, S. L., Ebbeck, V., and Weiss, M. R.: Fair Play for Kids: effects on the moral development of children in physical educarion. Research Quarterly Exercise Sport 66 (1995) 247.

[11] Huber, E. G.: Aufgaben und Bedeutung kindersportärztlicher Untersuchungen. Pädiat. Pädologie 10 (1975) 342.

[12] Kelder, S. H., Perry, C. L., Klepp, K. I., and Lytle, L. L.: Longitudinal tracking of adolescent smoking, physical activity, and food choice behaviors. Amer. J. Public Health 84 (1994) 1121.

[13] DiNubile, N. A.: Youth fitness - problems and solutions. Prev. Med. 22 (1993) 589.

[14] Dietz jr., W.H., and Gortmaker, S. L.: Do we fatten our children at the television set? Obesity and television viewing in children and adolescents. Pediatrics 75 (1985) 807.

[15] Kuntzleman, C. T.: Childhood fitness: what ist happering? What needs to be done? Prev. Med 22 (1993) 520.

[16] Schlicker, S. A., Borra, S. T., and Regan, C.: The weight and fitness status of United States children. Nutr. Reviews 52 (1994) 11.

[17] Harsha, D. W.: The benefits of physical activity in childhood. Amer. J. Med. Sciences 310 (1995) Suppl 1., 109.

[18] Edmundson, E., Parcel, G.S., Feldman, H. A., Elder, J., Perry, C. L., Johnson, C. C., Williston, B. J.. Stone, E. J., Yang, M., Lytle, L. L., and Webber, L.: The effects of the child and adolescent trial for cardiovascular health upon psychosocial determinants of diet and physical activity behavior. Prev. Med. 25 (1996) 442.

[19] Myers, L., Strikmiller, P. K., Webber, L. S., and Berenson, G. S.: Physical and sedentary activity in schoolchildren grades 5-8: the Boyalusa Heart Study. Med. Science Sports Exercise 28 (1996) 853.

[20] Kelder, S. H., Perry, C. L., Klepp, K. I., and Lytle, L. L.: Community-wide youth exercise promotion: Long-term outcomes of the Min nesota Heart Health Program and the class of 1989 study. J. School Health 63 (1993) 218.

[21] Fuchs, W.: Sport und Sportverein. [n: Jugendliche und Erwacnsene "85. Generationen im Vergleich. Leverkusen: Leske und Budrich (1985).

[22] Gesundheitsverhalten im Kindes- und Jugendalter: Ausgewählte Ergebnisse der Berlin-Bremen-Studie. Band 5 der Schriftenreihe des Bundesministeriums für Gesund heit. Baden-Baden: Nomos (1991).

[23] Heinemann, L.: Gesundheitsförderung im Kindes- und Jugendalter in Ostdeutschland, unveröffentlichtes Manuskript (1993)

[24] Huppertz, N., und Kraus, J.: Jugendliche in ihrer Freizeit - welche Veränderungen se. hen wir? Neue Praxis, Heft 2, (1995) 196.

[25] Washburn, R. A., Goldfield, S. R. W., Srrith, K. W., and McKinlay, J. B.: The validity of self-reported exercise-induced swearing as a measure of physical activiry. Amer. J. Epide. miol. 132 (1990) 107.

[26] La Porte, R. D., Black-Sandler, R., and Cauley, J. A.: The assessment of physical activity in older women: analysis of the interrelarionship and reliability of activity monitoring, activity surveys and caloric intake. J. Gerontol. 38 (1983) 394

[27] Albanes, D., Conway, J. M., Taylor, P. R., Moe, P. W., and Judd, J.: Validation and comparison of eight physical activity questionnaires. Epidemiology 1 (1990) 65

[28] Fuchs, R.: Messung der sportlichen Aktivität. In: Abschlußberichr Gesundheit im Kindesund Jugendalter, Kapitel 18. Unveröffentlichtes Manuskripr (1987).

[29] Taylor, H. L., Jacobs, D. R., Schucker, B., Knudsen, J,, Leon, A. S., and Debacker, G.: A questionnaire for the assessment of leisure time physical activities. J. chron. diseases 31 (1978) 741 .

[30] McArdle, W. D., Katch, F. L., and Katch, V. L.: Exercise physiology: energy, nutrition, and human performance. Philadelphia: Lea $\&$ Febiger (1981).

[31] Folsom, A. R., Caspersen, C. J., Taylor, H. L., Jacobs, D. R., Luepker, R. V., Gomez-Marin, O., Gillum, R. F., and Blackburn, H.: Leisure time physical activity and its relationship to coronary risk factors in a population-based sample. Amer. J. Epidemiol. 121 (1985) 570.

[32] Pate, R. R., Pratt, M., Blair, S. N., Haskell, W. L., Macera, C. A., Bouchard, C., Buchner, D., Ettinger, W., Hearh, G. W., King, A.C., Kriska, A., Leon, A. S., Marcus, B. H., Morris, J., Paffenbarger jr., R. S., Patrick, K., Pollock, M. L., Rippe, J. M., Sallis, J., and Wilmore, J. H.: Physical acriviry and public health. A recommendation from the centers for discase control and prevention and the American college of sports medicine. J. Amer. Med. Assoc. 273 (1995) 402.

[33] Anderssen, 1., Kiepp, K. I., Aas, H., and Jakobsen, R.: Stability in physical activity levels in young adolescents. Europ. J. Public Health 4 (1994) 175

[34] Röthlisberger, C., und Clamonte, R.: Sportliche Aktivität, personale Ressourcen und $\mathrm{Be}$ lastungen von Adoleszenten. Zeitschr. Gesundheitspsychol. Bd III (1995) 209-223.

[35] Lang, H. P., und Fuchs, R.: Prävalenz der sportlichen Aktivität: Forschungsstand. In: Abschlußbericht Gesundheit im Kindes- und Jugendalter, Kapitel 5. Unveröffentlichtes Manuskript (1987).

[36] Nader, P. R., Sellers, D. E., Johnson, C. C., Perry, C. L., Stone, E. J., Cook, K. C., Bebchuk, J., and Luepker, R. V.: The effect of acult participation in a sciool-jased family intervention to improve children's diet and physical activity: The child and adolescent trial for cardiovascular health. Prev. Med. 25 (1996) 455.

[37] Stucky-Ropp, R. C., and DiLorenzo, T. M.: Determinants of exercise in children. Prev. Med. 22 (1993) 880.

[38] Craig, S., Goldberg, J., and Dietz, W. H.: Psychosocial correlares of physical activity among fifth and eight graders. Prev. Med. 25 (1996) 506.

[39] Reynolds, K. D., Killen, J. D., Bryson, S. W. Maron, D. J., Taylor, C. B., Maccoby, N., and Farquhar, J. W.: Psychosocial predictors of physical activity in adolescents. Prev. Med. 19 (1990) 541.

[40] Biddle, S.: Exercise and psychosocial health. Physical. Educ., Recreation \& Dance 66 (1995) 292.

[41] Bretschneider, W. D., und Kramer, H. J.: Sportliche Interessen bei Schülern und Jugendlichen. Berlin, München, Frankfurt/M (1978).

Anschrift der Verfasser:

Dr. Hans Hüttner, Reinhard Dortschy, Heike Heß, Dr. Heidrun Kahl, Prof.Dr. Konrad Tietze, Gabriela Röseler, Robert Koch-Institut, Nordufer 20, 13353 Berlin. 